Abanico Veterinario. Enero-Diciembre 2020; 10:1-11. http://dx.doi.org/10.21929/abavet2020.11

Artículo Original. Recibido: 30/01/2020. Aceptado: 03/06/2020. Publicado: 15/06/2020. Clave: 2020-9.

\title{
Sustitución de heno de alfalfa por hojas de ajo y su efecto en la fermentación ruminal in vitro
}

Substitution of garlic leaves to alfalfa hay and its effect on in vitro ruminal fermentation

$$
\begin{gathered}
\text { Torres-Fraga Karla }^{1 * \mid \mathrm{ID}}, \text { Páez-Lerma Jesús }^{2} \mathrm{ID}, \text { Pámanes-Carrasco Gerardo }^{3 \mathrm{ID}} \text {, } \\
\text { Herrera-Torres Esperanza }^{\mathrm{ID}}, \text { Carrete-Carreón Francisco }^{4 \mathrm{ID}}, \text { Murillo-Ortiz }^{\text {Manuel }}{ }^{\star * 4} \mathrm{ID}
\end{gathered}
$$

${ }^{1}$ Estudiante del Doctorado en Ciencias Agropecuarias y Forestales de la Universidad Juárez del Estado de Durango, México. ${ }^{2}$ Tecnológico Nacional de México, Instituto Tecnológico de Durango, México. ${ }^{3}$ nstituto de Silvicultura e Industria de la Madera, Universidad Juárez del Estado de Durango, México. ${ }^{4}$ Facultad de Medicina Veterinaria y Zootecnia-Universidad Juárez del Estado de Durango. México. *Autor responsable: Torres-Fraga Karla. ${ }^{* *}$ Autor de correspondencia: Murillo-Ortiz Manuel, Carretera Durango - Mezquital Km 11.5, C.P. 34307. 6181830274. Durango, Durango, México. karlatf12@hotmail.com, jpaez@itdurango.edu.mx, gerardo.pamanes@gmail.com, hetoes99@yahoo.com.mx, focc1928mx@yahoo.com, manuelmurillo906@gmail.com

\section{RESUMEN}

Esta investigación tuvo como objetivo evaluar la sustitución de heno de alfalfa por hojas de ajo en la producción de gas in vitro y metano, así como en los patrones de fermentación ruminal. Se evaluaron cuatro tratamientos: (T1) heno de alfalfa (50\%); (T2) heno de alfalfa (33\%) + hojas de ajo crudo (17\%); (T3) heno de alfalfa (17\%) + hojas de ajo crudo (33\%) y (T4) hojas de ajo crudo (50\%). Los valores más altos de "a" (producción de gas a partir de la fracción soluble); "b" (producción de gas a partir de la fracción insoluble) y "c" (tasa de producción de gas) se encontraron en T4 $(\mathrm{mL})$; mientras que fueron menores en T1 $(P<0.05)$; pero no se observaron diferencias entre T2 y T3 ( $\mathrm{P}>0.05)$. La concentración más alta de nitrógeno amoniacal (N-NH3) se registró en $\mathrm{T} 4$ y la más baja en $\mathrm{T} 1(\mathrm{P}<0.05)$. Las concentraciones de propionato aumentaron con T4 y disminuyeron con T1, mientras que las de acetato disminuyeron con T4 y aumentaron con T1 ( $\mathrm{P}<0.05)$. La producción de metano más alta se registró en T1 y la más baja en T4 ( $P$ $<0.05)$. Se concluye, que la sustitución de heno de alfalfa por hojas de ajo en dietas de bovinos carne, mejoró la fermentación ruminal y disminuyó la producción de metano en condiciones in vitro.

Palabras clave: hojas de ajo, producción de gas, fermentación ruminal y metano.

\begin{abstract}
This research aimed to evaluate the substitution of alfalfa hay to garlic leaves during the in vitro gas and methane production, as well as in ruminal fermentation patterns. There were four treatments: (T1) alfalfa hay (50\%); (T2) alfalfa hay (33\%) + raw garlic leaves (17\%); (T3) alfalfa hay $(17 \%)$ + raw garlic leaves (33\%) and (T4) raw garlic leaves (50\%). The highest values of "a" (gas production from the soluble fraction); "b" (gas production from the insoluble fraction) and "c" (gas production rate) were found in $\mathrm{T} 4(\mathrm{~mL})$; while lower in $\mathrm{T} 1(\mathrm{P}<0.05)$; but no differences were
\end{abstract}


observed among T2 and T3 ( $>>0.05)$. The highest ammonia-nitrogen (NH3-N) concentration was recorded in $\mathrm{T} 4$ and the lowest in $\mathrm{T} 1(\mathrm{P}<0.05)$. The propionate concentrations increased with $\mathrm{T} 4$ and decreased with $\mathrm{T} 1$, whereas the acetate decreased with $\mathrm{T} 4$ and increased with $\mathrm{T} 1(\mathrm{P}<0.05)$. The highest methane production was recorded in $\mathrm{T} 1$ and the lowest in $\mathrm{T} 4(\mathrm{P}<0.05)$. It is concluded that substitution of alfalfa hay to garlic leaves in beef cattle diets, improved the ruminal fermentation and decreased methane production under in vitro conditions.

Keywords: garlic leaves, gas production, ruminal fermentation, methane.

\section{INTRODUCCIÓN}

Hay una tendencia en la demanda a nivel mundial de consumo de ajo, lo que ha generado el empleo en la alimentación, particularmente de rumiantes, de sus principales subproductos como cáscaras, hojas y pajas (Kallel y Ellouz, 2017). En diversos países las hojas de ajo que se obtienen durante la cosecha son incineradas, y como consecuencia surgen problemas asociados con los costos de eliminación y contaminación ambiental (Han et al., 2013).

En este sentido, se puede decir que las emisiones de metano por los rumiantes no solo causan serios problemas ambientales, sino también representa una fuente importante de pérdida de energía para los animales. (Gallegos et al., 2019; Lan y Yang, 2019). La producción de gases de efecto invernadero (GEI) que se producen en los diferentes sistemas ganaderos y su impacto en los cambios climáticos, son una preocupación importante en todo el mundo. Se ha informado que el metano entérico es el GEI más importante emitido (50-60\%), en los sistemas de producción de rumiantes (Tapio et al., 2017; Haque, 2018).

Como resultado de esta problemática, se han hecho muchos intentos para modificar la fermentación ruminal y disminuir la producción de metano; entre ellos se encuentra la incorporación a las dietas de rumiantes de forrajes de buena calidad, como el heno de alfalfa. También se ha demostrado que el ajo y algunos de sus componentes disminuyen la producción de metano en condiciones in vivo e in vitro (Kamra et al., 2012). De hecho, hasta ahora pocos estudios han investigado los efectos del ajo y sus subproductos de cosecha sobre la fermentación ruminal y la producción de metano.

En función de lo anterior, nosotros asumimos que la sustitución de hojas de ajo por heno de alfalfa en dietas de bovinos carne, produce cambios similares en los patrones de fermentación ruminal y producción de metano durante la fermentación in vitro. Por lo tanto, el objetivo del presente trabajo fue evaluar la sustitución de heno de alfalfa por hojas de ajo en la producción de gas in vitro y metano, así como en los patrones de fermentación ruminal.

\section{MATERIAL Y MÉTODOS}

El experimento se realizó en la unidad metabólica y en el laboratorio de Nutrición Animal de la Facultad de Medicina Veterinaria y Zootecnia de la Universidad Juárez del estado de Durango (México). 
Muestreo de hojas de ajo crudo. Las muestras (hojas con $25 \mathrm{~cm}$ de longitud) de ajo crudo (Allium sativum) utilizadas en este estudio, se recolectaron en la región norte de México. Para garantizar un muestreo representativo, las muestras se recolectaron cinco veces, entre enero y mayo del año 2019. Antes del análisis químico y de los ensayos in vitro, las muestras se secaron y luego se molieron a través de una malla de $1 \mathrm{~mm}$.

Tratamientos experimentales. En cada tratamiento experimental se utilizó como fuente de forraje heno de alfalfa y heno de hojas de ajo crudo. La composición química del heno de alfalfa y de heno de hojas de ajo crudo se muestra en la tabla 1.

Tabla 1. Composición química del heno de alfalfa y de las hojas de ajo (MS \%)

\begin{tabular}{llc}
\hline & Heno de alfalfa & Hojas de ajo \\
\hline MS & 90.2 & 89.5 \\
MO & 88.9 & 90.1 \\
PC & 18.0 & 18.1 \\
EE & 1.6 & 2.1 \\
FDN & 33.2 & 35.1 \\
FDA & 23.7 & 26.7 \\
CHT & 68.8 & 66.3 \\
CNF & 35.6 & 31.2 \\
L & 5.7 & 6.8
\end{tabular}

$\mathrm{MS}$ = materia seca; $\mathrm{MO}$ = materia orgánica; $\mathrm{PC}$ = proteína cruda; EE = extracto de etéreo; FDN = fibra de detergente neutro; FDA = fibra detergente ácida; $\mathrm{CHT}=$ carbohidratos totales, $\mathrm{CNF}=$ carbohidratos no fibrosos; $\mathrm{L}=$ lignina

Se evaluaron cuatro tratamientos: (T1) heno de alfalfa (50\% MS) + hojas de ajo crudo (0\% MS); (T2) heno de alfalfa (33\% MS) + hojas de ajo crudo (17\% MS); Heno de alfalfa T3 (17\% MS) + hojas de ajo crudo (33\% MS); T4 heno de alfalfa $(0 \% \mathrm{MS})+$ hojas de ajo crudo (50\% MS). En todos los tratamientos se utilizaron proporciones similares de maíz molido, semillas de algodón y minerales. La composición nutricional de los tratamientos experimentales se muestra en la tabla 2.

Composición nutricional. Cada muestra de los tratamientos experimentales, se analizó por triplicado para materia seca (MS), proteína cruda (PC), extracto de éter (EE) y materia orgánica (MO) (AOAC, 2000). Los análisis de fibra de detergente neutro (FDN), fibra de detergente ácido (FDA) y lignina en detergente ácido (ADL), se determinaron utilizando la técnica de bolsas filtros con un analizador de fibra (ANKOM Technology, Fairport, NY, EE. UU.). El contenido de carbohidratos no fibrosos (CNF), se calculó utilizando la diferencia entre el total de carbohidratos (CHT) y el \% de FDN. La digestibilidad in vitro de la materia seca (DIVMS) y de la materia orgánica (DIVMO), se determinó utilizando el incubador Daisy (ANKOM Technology, Fairport, NY, EE. UU.). 
Tabla 2. Composición nutricional de los tratamientos experimentales

\begin{tabular}{lcccc}
\hline & & & Tratamientos & \\
\hline Heno de alfalfa & T1 & T2 & T3 & 0 \\
Hojas de ajo & 50 & 33 & 17 & 50 \\
Maíz molido & 0 & 17 & 33 & 39 \\
Harinolina & 39 & 39 & 39 & 10 \\
Minerales & 10 & 10 & 10 & 1 \\
\hline & 1 & 1 & 1 & 93.9 \\
\hline MS & & Composición química (MS\%) & 93.0 \\
MO & 96.1 & 95.6 & 95.6 & 15.6 \\
PC & 92.5 & 91.8 & 92.0 & 2.1 \\
EE & 15.0 & 14.6 & 14.7 & 64.2 \\
DIVMS & 2.0 & 2.0 & 2.5 & 62.2 \\
DIVMO & 58.2 & 60.3 & 62.2 & 47.1 \\
FDN & 56.1 & 58.5 & 59.1 & 19.2 \\
FDA & 53.3 & 49.3 & 50.9 & 28.2 \\
CNF & 28.3 & 23.3 & 21.7 & 23.9 \\
\hline
\end{tabular}

$\mathrm{MS}$ = materia seca; $\mathrm{MO}$ = materia orgánica; $\mathrm{PC}$ = proteína cruda; $\mathrm{EE}$ = extracto de etéreo; DIVMS= digestibilidad in vitro de la materia seca, $\mathrm{DIVMO}=$ digestibilidad in vitro de la materia orgánica; FDN = fibra de detergente neutro; FDA = fibra detergente ácida; $C N F=$ carbohidratos no fibrosos

Gas in vitro, patrones de fermentación ruminal y producción de metano. La producción de gas in vitro se midió utilizando el sistema de producción de gas ANKOM. El líquido ruminal se recolectó aproximadamente $3 \mathrm{~h}$ después de la alimentación matutina de dos novillos fistulados en el rumen, alimentados a libre acceso con heno de alfalfa. El fluido ruminal se filtró inmediatamente a través de cuatro capas de gasa y se transportó al laboratorio en un termo sellado. El fluido ruminal resultante se purgó con $\mathrm{CO}_{2}$ antes de su uso como inóculo. Se pesaron por triplicado aproximadamente $1 \mathrm{~g}$ de muestras secas y molidas de cada tratamiento y se colocaron en módulos de vidrio. Se pipeteó en cada módulo líquido ruminal y se mezcló con saliva de McDougall (120 mL).

La producción de gas se registró después de 2, 4, 6, 8, 12, 16, 24, 36, 48, 72 y 96 h de incubación. La cinética de producción de gas (PD) se ajustó al modelo no lineal propuesto por Ørskov y McDonald (1979), el cual es el siguiente: PD (t) = a+b×(1-exp-ct); donde "a" es la producción de gas a partir de la fracción soluble $(\mathrm{mL})$, "b" es la producción de gas a partir de la fracción insoluble, $(\mathrm{mL})$, "c" es la tasa constante de producción de gas $(\mathrm{mL} / \mathrm{h})$ $y$ " $t$ " es el tiempo de incubación.

Para la determinación de metano, se recolectaron después de 24 h de incubación, $10 \mathrm{~mL}$ de gas del espacio superior de los módulos del sistema de producción de gas, de los cuales se tomaron submuestras de $3 \mathrm{~mL}$ y enseguida se determinó la producción de metano en un cromatógrafo de gases (Kara, 2015).

Asimismo, después de la finalización de la incubación de las 24 h y antes de la filtración, se recolectaron dos muestras $(5 \mathrm{~mL})$ del líquido de cada módulo, e inmediatamente se 
midió el $\mathrm{pH}$ y se almacenaron a $-20^{\circ} \mathrm{C}$ hasta el posterior análisis de nitrógeno amoniacal (N-NH3) y ácidos grasos volátiles (AGV) (Ammar et al., 2005).

Análisis estadístico. Se realizó un análisis de varianza para un diseño completamente al azar, para comparar los parámetros producción de gas in vitro, metano y patrones fermentación ruminal con el procedimiento MIXED de SAS (2002). Las medias individuales se separaron mediante la prueba de rango múltiple de Tukey. Las diferencias de medias se consideraron significativas a $\mathrm{P}<0.05$.

\section{RESULTADOS Y DISCUSIÓN}

Parámetros de producción de gas in vitro. Los valores más altos de "a", "b" y "c" (tabla $3)$, se encontraron en T4; mientras que los valores más bajos en T1 ( $P<0.05)$; pero no se observaron diferencias entre T2 y T3 (P> 0.05). Se observaron diferencias en el valor " $a+b$ " entre tratamientos $(P<0.05)$. Los resultados son consistentes con los reportados por Tag El-Dini et al. (2012), quienes encontraron un valor "a" de $0.60 \%$ cuando se fermentó en condiciones in vitro pulpa de ajo a un nivel del $3 \%$ del concentrado.

En este estudio, las diferencias observadas entre tratamientos en el valor "a" pueden atribuirse a las diferentes concentraciones de los tratamientos en carbohidratos no fibrosos (La $\mathrm{O}$ et al., 2008). Los valores promedio de "b" y a + b encontrados en el presente estudio fueron $116.0 \mathrm{~mL} 200 \mathrm{mg}^{-1} \mathrm{MS}$ y $116.5 \mathrm{~mL} 200 \mathrm{mg}^{-1} \mathrm{MS}$, respectivamente. Estos valores coinciden con los informados por Sahli et al. (2018) cuando se incubaron in vitro ajo en polvo, en dosis de $32 \mathrm{mg}$, en una dieta compuesta de $50 \%$ de heno de ryegrass y $50 \%$ de concentrado comercial.

El valor "c" registrado con T4 $\left(9.2 \mathrm{~mL} \mathrm{~h}^{-1}\right)$ fue superior al encontrado por Anassori et al. (2012) (3.5 $\left.\mathrm{mL} \mathrm{h}^{-1}\right)$, quienes evaluaron la cinética de producción de gas in vitro de bulbo de ajo crudo.

En el estudio actual, la alta tasa constante de producción de gas "c" registrada en T4, indica una alta disponibilidad de nutrientes para los microorganismos ruminales; mientras que los valores "c" más bajos observados en T1 podrían ser el resultado de un alto contenido de FDN, cuyos componentes químicos podrían disminuir la velocidad de fermentación del sustrato (Fievez et al., 2005).

Tabla 3. Parámetros de producción de gas de los tratamientos experimentales

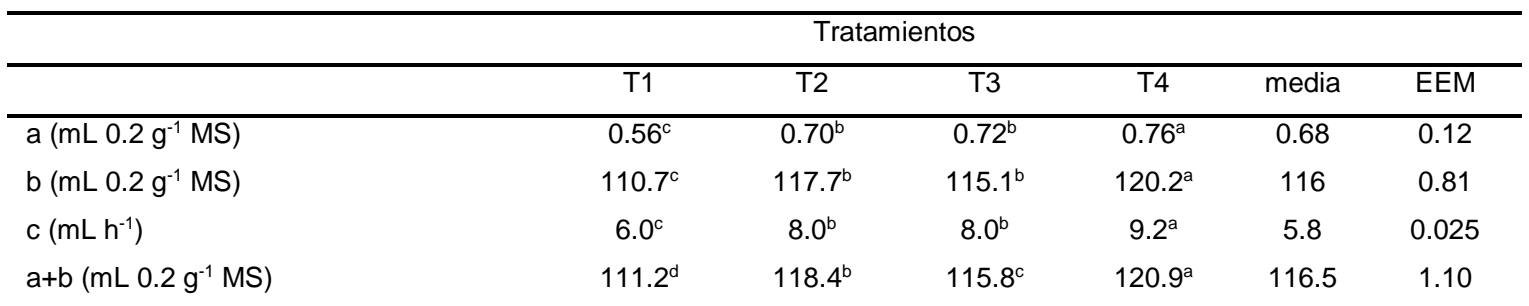

${ }^{a b c}$ Los valores con letras diferentes en la misma fila son estadísticamente diferentes $(P<0.05)$.

$\mathrm{a}=$ Producción de gas a partir de la fracción soluble, $\mathrm{b}=$ Producción de gas a partir de la fracción insoluble; $\mathrm{c}=$ Tasa constante de producción de gas; $\mathrm{a}+\mathrm{b}=$ Producción potencial de gas; EEM: Error estándar de la media 
Patrones de fermentación ruminal y producción de metano. Los patrones de fermentación ruminal y la producción de metano se presentan en la Tabla 4. La concentración de N-NH3 varió de 15.1 a $18.1 \mathrm{mg} / \mathrm{dL}$. La concentración más alta de $\mathrm{N}$ $\mathrm{NH} 3$ se registró en T4 y la más baja en $\mathrm{T} 1(\mathrm{P}<0.05)$. No hubo diferencias entre T2, T3 y T4 en las concentraciones de AGV totales $(P>0.05)$; pero ambos tratamientos fueron diferentes a T1 $(P<0.05)$.

La concentración de acetato más alta se registró en T1 y la más baja en T4 $(P<0.05)$; mientras que la concentración más alta de propionato se registró en T4 y la más baja en T1 $(P<0.05)$. No hubo diferencia entre T1, T2 y T3 en la relación acetato:propionato $(P>$ $0.05)$; pero ambos tratamientos fueron diferentes a T4 $(P<0.05)$. La producción de metano más alta se registró en T1 y la más baja en T4 $(P<0.05)$.

Las concentraciones ruminales de $\mathrm{N}-\mathrm{NH} 3$ registradas en todos los tratamientos evaluados, se mantuvieron dentro del rango sugerido para un óptimo crecimiento microbiano. Según Wanapat y Pimpa (1999) un rango de 15 a $30 \mathrm{mg} / 100 \mathrm{~mL}$ son las concentraciones mínimas requeridas para una síntesis óptima de proteínas microbianas. En contraste, con los resultados obtenidos en este estudio, varios estudios in vitro e in vivo informan que el aceite de ajo y el polvo de ajo reducen o no tienen efecto sobre la concentración de N-NH3 en el fluido ruminal (Cardozo et al., 2004); sin embargo, Yang et al. (2007) encontraron un aumento en la concentración de N-NH3 ruminal, cuando se incorporó ajo crudo a dietas de vacas lactantes. En este estudio, la mayor concentración de N-NH3 obtenida con T4 podría explicarse por el alto contenido de proteína cruda que suministran las hojas de ajo (Panthee et al., 2017).

Tabla 4. Patrones de fermentación ruminal y producción de metano de los tratamientos experimentales

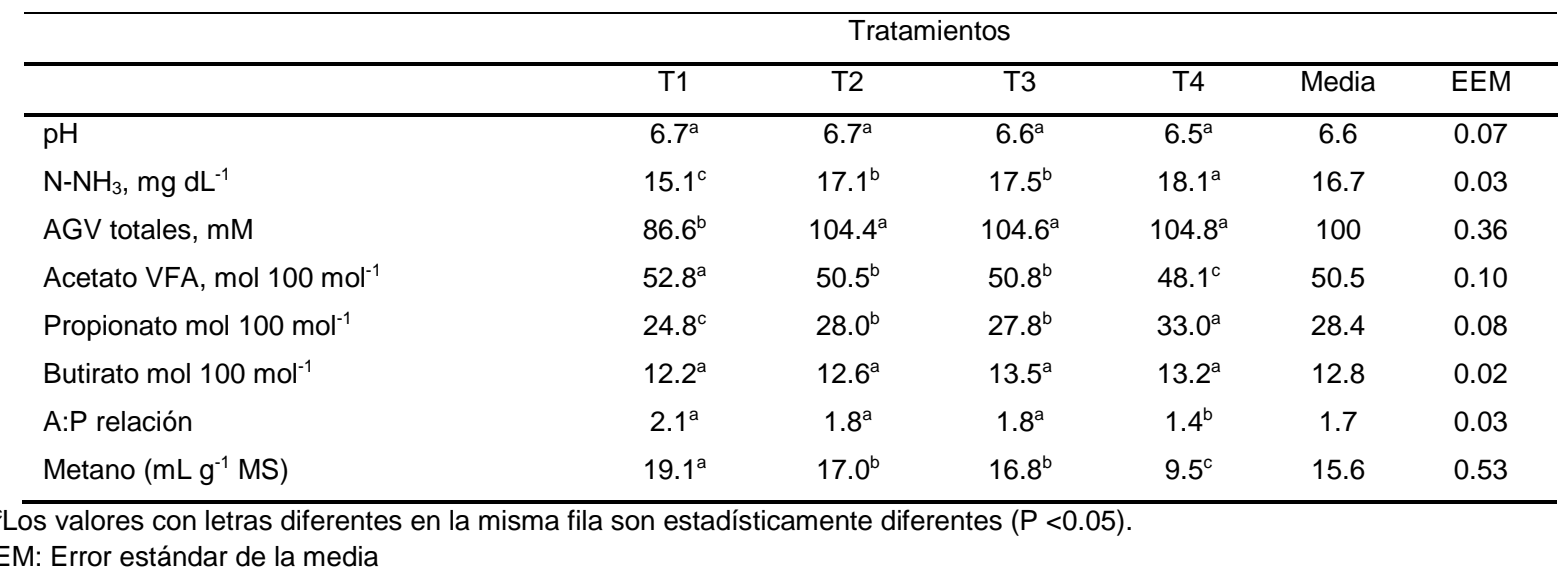

Las concentraciones totales de AGV obtenidas con T2, T3 y T4 son consistentes con otros estudios in vitro, donde no difirieron con la adición de aceites de ajo (Klevenhusen et al., 2011). La concentración de propionato observada en T4 fue mayor que en los otros tratamientos; esto se evidencía con la disminución de la proporción acetato:propionato. 
Además, el aumento en la concentración de propionato podría atribuirse a la cantidad de carbohidratos no fibrosos suministrados por T4 (Van Soest, 1994). De igual manera, la reducción en la proporción de acetato a propionato registrada en T4, indica una mejora en el aporte de energía neta (Zhong et al., 2019). Resultados similares son reportados Mirzaei-Aghsaghali y Maheri-Sis (2011), quienes encontraron que el aceite de ajo aumentó las proporciones de propionato y butirato, pero redujo la proporción de acetato. En este estudio, la baja concentración de metano se obtuvo con T4 (alto en hojas de ajo); y coincide con los resultados encontrados por Kongmun et al., (2010), quienes informan una disminución en la producción de metano cuando se evaluó el ajo en polvo en las dietas de rumiantes. En nuestro estudio, los valores de metano registrados en los cuatro tratamientos, fueron inferiores a los reportados por Zafarian y Manafi (2013), quienes evaluaron en condiciones in vitro, la adición de diferentes dosis de ajo en polvo (2, 4 y $6 \% \mathrm{MS}$ ) en dietas con un $50 \%$ de fibra y un $50 \%$ de concentrado.

En este estudio, la reducción en la producción de metano registrada en T4 en comparación con los otros tratamientos, podría atribuirse al aumento en la concentración de propionato, debido a que la formación de propionato consume equivalentes reductores; mientras que la formación de acetato genera $\mathrm{H} 2$ para la metanogénesis (Moss et al., 2000). Cualquier componente o variable de la dieta que cause un cambio a favor de la producción de propionato irá acompañado de una reducción en la producción de metano por unidad de dieta fermentada; mientras que se observa lo contrario para el acetato y el butirato (Pinares-Patiño, 2003). Sin embargo, las diferencias observadas en este estudio en la producción de metano, en comparación con las reportadas en la literatura, pueden estar relacionadas con diferentes variedades genéticas y posiblemente con el estado fenológico de la planta de ajo; lo que resulta en diferentes contenidos de la pared celular y como consecuencia en los patrones de fermentación ruminal (Lee et al., 2017).

\section{CONCLUSIONES}

La sustitución de heno de alfalfa por hojas de ajo en dietas de bovinos carne, mejoró algunos indicadores de importancia nutricional, como la producción de gas a partir de la fracción soluble y la tasa constante de producción de gas. De igual manera, incrementó las concentraciones de N-NH3 y AGV totales y redujo la producción de metano. Se necesitan investigaciones adicionales para evaluar los efectos de las hojas de ajo en las dietas de rumiantes sobre los patrones de fermentación ruminal y la producción de metano en condiciones in vivo; así como para evaluar la sostenibilidad de la suplementación de hojas de ajo para mitigar la metanogénesis del rumen sin efectos perjudiciales en el rendimiento animal. 


\section{LITERATURA CITADA}

AMMAR H, López S, González JS. 2005. Assessment of the digestibility of some Mediterranean shrubs by in vitro techniques. Animal Feed Science and Technology. 119: 323-331. https://doi.org/10.1016/j.anifeedsci.2004.12.013

ANASSORI E, Dalir-Naghadeh B, Pirmohammadi R, Taghizadeh A, Asri-Rezaei S, Farahmand-Azar S, Besharati M, Tahmoozi M. 2012. In vitro assessment of the digestibility of forage based sheep diet, supplemented with raw garlic, garlic oil and monensin. Veterinary Research Forum. 3: 5-11. https://www.ncbi.nlm.nih.gov/pmc/articles/PMC4312812/

AOAC. 2000. Official Methods of Analysis, Association of AOAC International. 17th Ed. Association of Official Analytical Chemists, Arlington, USA. Disponible: https://www.aoac.org/official-methods-of-analysis-21st-edition-2019/

CARDOZO P W, Calsamiglia S, Ferret A, Kamel C. 2004. Effects of natural plant extracts on ruminal protein degradation and fermentation profiles in continuous culture. Journal of Animal Science. 82:(11) 3230-3236.

https://doi.org/10.2527/2004.82113230x

FIEVEZ V, Babayemi OJ, Demeyer D. 2005. Estimation of direct and indirect gas production in syringes: A tool to estimate short chain fatty acid production that requires minimal laboratory facilities. Animal Feed Science and Technology. 123-124: 197-210. https://doi.org/10.1016/j.anifeedsci.2005.05.001

GALLEGOS FP, Bañuelos VR, Delgadillo RL, Echavarría CF, Meza LC, Rodríguez TD. 2019. Differential evaluation of oregano extracts in the production of volatile fatty acids and methane during ruminal fermentation in vitro. Abanico Veterinario. 9(1):1-18. http://dx.doi.org/10.21929/abavet2019.91

HAN X, Cheng Z, Meng H, Yang X, Ahmad I. 2013. Allelopathic effect of decomposed garlic (Allium sativum L.) stalk on lettuce (L. Sativa Var. Crispa L.). Pakistan Journal of Botany. 45:225-233. https://www.pakbs.org/pjbot/PDFs/45(1)/32.pdf

HAQUE MN. 2018. Dietary manipulation: a sustainable way to mitigate methane emissions from Ruminants. Journal Animal Science and Technology. 60: 15. https://doi.org/10.1186/s40781-018-0175-7 
KALLEL F, Ellouz CS. 2017. Perspective of garlic processing wastes as low-cost substrates for production of high-added value products: a review. Environmental Progress and Sustainable Energy. 36: 1765-1777. https://doi.org/10.1002/ep.12649

KAMRA DN, Agarwal N, Sakthivel PC, Chaudhary LC. 2012. Garlic as a rumen modifier for eco-friendly and economic livestock production. Journal of Applied Animal Research. 40: 90 96. https://doi.org/10.1080/09712119.2011.607764

KARA K. 2015. In vitro methane production and quality of corn silage treated with maleic acid. Italian Journal of Animal Science. 14: 718-722. https://doi.org/10.4081/ijas.2015.3994

KLEVENHUSEN F, Zeitz JO, Duval S, Kreuzer M, Soliva CR. 2011. Garlic oil and its principal component diallyl disulfide fail to mitigate methane, but improve digestibility in sheep. Animal Feed Science and Technology. 166-167: 356-363. https://doi.org/10.1016/j.anifeedsci.2011.04.071

KONGMUN P, Wanapat M, Pakdee P, Navanukraw C. 2010. Effect of coconut oil and garlic powder on in vitro fermentation using gas production technique. Livestock Science. 127: 38-44. https://doi.org/10.1016/j.livsci.2009.08.008

LA O, García R, Ruiz O, Castillo Y, Muro A, Rodríguez C, Arzola C, González H, Ortiz B. 2008. Potencial fermentativo ruminal in vitro de dos árboles (Pithecellobium dulce y Tamarindos indica) de importancia para la ganadería en ecosistemas frágiles, salinos y de alta sequía, situados en el Oriente de Cuba. Revista Cubana de Ciencia Agrícola. 42: 57-61. https://www.redalyc. org/articulo.oa?id=193015413009

LAN W, Yang C. 2019. Ruminal methane production: Associated microorganisms and the potential of applying hydrogen-utilizing bacteria for mitigation. Science of the Total Environment. 654: 1270-1283. https://doi.org/10.1016/j.scitotenv.2018.11.180

LEE YH, Kim YI, Oh YK, Ahmadi F, Kwak WS. 2017.Yield survey and nutritional evaluation of garlic stalk for ruminant feed. Journal of Animal Science and Technology. 59: 22. https://doi.org/10.1186/s40781-017-0147-3

MIRZAEI-AGHSAGHALI A, Maheri-Sis N. 2011. Factors affecting mitigation of methane emission from ruminants I: Feeding strategies. Asian Journal of Animal and Veterinary Advances. 6:888-908. http://dx.doi.org/10.14269/2318-1265/jabb.v4n1p22-31 
MOSS AR, Jouany JP, Newbold J. 2000. Methane production by ruminants: its contribution to global warming. Annals of Zootechnie. 49: 231-253. https://doi.org/10.1051/animres:2000119

ØRSKOV ER, McDonald I. 1979. The estimation of protein degradability in the rumen from incubation measurements weighted according to rate of passage. Journal of Agricultural Science. 92: 499-503. https://www.cambridge.org/core/services/aop-cambridgecore/content/view/E2DB4F2290E374E10E9800E512D127A7/S0021859600063048a.pdf lestimation_of_protein_degradability_in_the_rumen_from_incubation_measurements_w eighted_according_to_rate_of_passage.pdf

PANTHEE A, Matsuno A, Al-Mamun M, Sano H. 2017. Effect of feeding garlic leaves on rumen fermentation, methane emission, plasma glucose kinetics, and nitrogen utilization in sheep. Journal of Animal Science and Technology. 59: 14. https://doi.org/10.1186/s40781-017-0139-3

PINARES-PATIÑO CS, Ulyatt MJ, Waghorn GC, Lassey KR, Barry TN, Holmes CW, Johnson DE. 2003. Methane emissions by alpaca and sheep fed on lucerne hay or grazed on pastures of perennial ryegrass/white clover or birdsfoot trefoil. Journal of Agricultural Science. 140: 215-226. https://doi.org/10.1017/S002185960300306X

SAHLI F, Darej C, Moujahed N. 2018. Potential of white garlic powder (Allium sativum L.) to modify in vitro ruminal fermentation. South African Journal of Animal Science. 48: 253260. http://dx.doi.org/10.4314/sajas.v48i2.6

SAS. System Analytics Static. 2002. User's Guide: Statistics Ver 9.0. SAS Inst. Inc., Cary, NC, USA.

TAG EL-Dini AE, Mohara MS, Nour AA, Nasser ME. 2012. Effect of some herbs on the rumen fermentation: 1- Effect of ginger (zingiber officinale) and garlic (allium sativum) on gas production, energy values, organic matter digestibility and methane emission, in vitro. Journal Agriculture and Enviromental Science, Damanhoure University Egypt. 11: 33-52. http://www.damanhour.edu.eg/pdf/agrfac/Root1/Vol.11-2-2.pdf

TAPIO I, Snelling TJ, Strozzi F, Wallace RJ. 2017. The ruminal microbiome associated with methane emissions from ruminant livestock. Journal of Animal Science and Biotechnology. 8: 7. https://doi.org/10.1186/s40104-017-0141-0 
VAN Soest PJ. 1994. Nutritional Ecology of the Ruminant. Ithaca, Cornell University Press, USA.

https://books.google.es/books?hl=es\&lr=\&id=TlluDwAAQBAJ\&oi=fnd\&pg=PP1\&dq=Van +Soest+P+J.+(1994).+Nutritional+Ecology+of+the+Ruminant.+Ithaca,+Cornell+Universit $\mathrm{y}+$ Press, + USA.\&ots=InCgEloYfy\&sig=KEkNXOzwc9BFqFueYB25IK5Hvk8\#v=onepage\& $q \& f=f a l s e$

WANAPAT M, Pimpa O. 1999. Effect of ruminal $\mathrm{NH}_{3}-\mathrm{N}$ levels on ruminal fermentation, purine derivatives, digestibility and rice straw intake in swamp buffaloes. AsianAustralasian Journal of Animal Sciences. 12: 904-907. https://doi.org/10.5713/ajas.1999.904

YANG WZ, Benchaar C, Ametaj BN, Chaves AV, He ML, McAllister TA. 2007. Effects of garlic and juniper berry essential oils on ruminal fermentation and on the site and extent of digestion in lactating cows. Journal of Dairy Science. 90: 5671-5681. https://doi.org/10.3168/jds.2007-0369

ZAFARIAN R, Manafi M. 2013. Effect of Garlic Powder on Methane Production, Rumen Fermentation and Milk Production of Buffaloes. Annual Review and Research in Biology. 3:1013-1019. http://www.journalarrb.com/index.php/ARRB/article/view/24989

ZHONG RZ, Xiang H, Cheng L, Zhao C, Wang F, Zhao X, Fang Y. 2019. Effects of feeding garlic powder on growth performance, rumen fermentation, and the health status of lambs infected by gastrointestinal nematodes. Animals. 9: 2-10.

https://doi.org/10.3390/ani9030102 